\title{
Generating controlled localized stimulations on haptic displays by modal superimposition
}

\author{
Ehsan Enferad $^{\mathrm{a}}$, Christophe Giraud-Audine ${ }^{\mathrm{b}, *}$, Frédéric Giraud ${ }^{\mathrm{a}}$, Michel \\ Amberg $^{\mathrm{a}}$, Betty Lemaire Semail ${ }^{\mathrm{a}}$ \\ ${ }^{a}$ Université de Lille, EA 2697 - L2EP - Laboratoire d'Electrotechnique et d'Electronique \\ de Puissance, F-59000 Lille, France \\ ${ }^{b}$ Arts et Métiers Paris Tech, EA 2697 - L2EP - Laboratoire d'Electrotechnique et \\ d'Electronique de Puissance, F-59000 Lille, France
}

\begin{abstract}
In the field of tactile feedback, researchers try to generate localized stimulations on screens. Some solutions such as time reversal or phased array use vibration induced in the screen equipped with piezoelectric ceramics. We propose to use the modal basis to reproduce a specified velocity field on such devices. We explain the theory and propose a methodology to practically synthesize the voltages to achieved a controlled focusing in a given time. Experiments on a simple demonstrator are in good agreement with the theory for various velocity fields and a reduced number of modes.
\end{abstract}

Keywords: Modal Analysis, Modal synthesis, Human Machine Interaction, Haptic, Focusing

\section{Introduction}

Tactile displays are now ubiquitous, the most common example being smartphones or tablets. They also have a great potential to realize virtual control panel for e.g. machine tools or automotive applications. However, one important limitation is that the main feedback channel toward the user is visual. Tactile or auditive feedback are at the moment crude, although it is commonly admitted and demonstrated that multiple sensory channels

\footnotetext{
${ }^{*}$ Corresponding author. Arts et Métiers Paris Tech CER de Lille, 8 Bd Louis XIV, 59000 Lille

Email address: christophe.giraud-audine@ensam.eu (Christophe Giraud-Audine)
} 
can enhance task performances and usability $[1,2]$. Hence, there are active researches to reduce the cognitive overload induced by visual confirmation during human machine interaction by stimulating the fingertip [3]. In this paper, a solution that can be simply implemented on a screen is examined. Unlike pin array technologies which require specific designs [4], friction modulation is an approach where, by some mechanical action, the friction coefficient perceived by the user is modified. Two main techniques fall into this category: electro-vibration (EV) and vibro-tactile (VT) devices.

Electro-vibration uses electrostatic force to attract the skin $[5,6]$ or a slider [7] on a surface submitted to a high voltage. Since the normal force at the contact area is modified $[8,9]$, the friction force is modulated simultaneously. This technology is versatile, however the effect can be mitigated by environmental conditions such as moisture [10].

Vibro-tactile is already widely used in current devices where the vibrations produced by an eccentric-mass motor convey basic pieces of information. Elastic waves are well suited to realize tactile feedback, they require minimal modification thanks to the use of piezoelectric patches, and can provide interaction at distance. Hence, more sophisticated approaches have been proposed to render high fidelity vibrations. Out of plane vibrations can produce friction modulation due to intermittent contact [11] and squeeze film effect $[12,13,14]$. Combination of in plane and out of plane vibration can also be used to increase the velocity at the screen/finger interface $[15,16]$. Usually, resonance is taken advantage of to reduce the power requirement and achieve usable displacement. Therefore, the whole surface is active, and to render textures, the vibration must be modulated with respect to the finger position $[17,18,19]$. By principle, these techniques are not suited for local stimulation or to stimulate several fingers independently. Besides, as it relies on the friction coefficient, the finger must move on the surface.

To address this shortcoming, phased array and time reversal of elastic waves have been proposed to achieve localized tactile stimulation [20, 21]. Unlike the previous techniques, elastic waves are propagated in the screen before interfering constructively at given time and position. To do so, the transducers composing phased arrays produce the same vibration delayed by a time interval proportional to the distance separating them from the focus target. Time reversal allows to improve the focusing accuracy and resolution $[22,23]$. This implies an additional knowledge resulting from an identification procedure. One limitation is that the focus pattern and its transient are not controlled, and external parameters can mitigate the effects [24]. As a matter of fact, this brief overview shows that the two last techniques actually rely on similar devices, and so they could be combined. To address 
the robustness issue, we propose an alternative approach to localized stimulation that can be combined with friction control. It is based on a truncated modal decomposition to design a focusing shape and control its position as suggested in [21]. This paper is in the continuity of our previous work that demonstrated that the resulting focus was viable to generate stimuli [25]. First we recall the basic theory that links the piezoelectric patches placement to the vibration problem then the methodology to specify the time evolution. Optimal voltage distribution to avoid saturation is also discussed. In the next part, we demonstrate the validity through various experiments and address some specific issues. Finally the last sections are devoted to discussion and conclusion.

\section{Operation principle}

\subsection{Assumptions}

In order to illustrate the idea presented, a special case is considered, but the method actually uses the orthogonality of the elastic vibration mode shapes [26] and therefore it can be generalized to other geometries. Since the point of this section is to explain how several modes are excited simultaneously in a controlled manner, a simple model involving a rectangular plate (the substrate) with piezoelectric patches glued symmetrically (bimorph regions) is considered.

The volume occupied by the substrate is $\Omega$ and its surface in the mid-plane is $\Gamma$. The $N_{b}$ bimorph domains will be denoted $\Omega_{i}$ and $\Gamma_{i}\left(i \in\left\{1 \ldots N_{b}\right\}\right)$ respectively for their volume and their surface in the mid-plane. Finally, the remaining substrate without patches is in $\Omega_{0}=\Omega \backslash \cup_{i=1}^{N_{b}} \Omega_{i}$, defining the

set $\Gamma_{0}=\Gamma \backslash \cup_{i=1}^{N_{b}} \Gamma_{i}$ as it intercept the mid-plane. For each sub-domain, we consider a Kirchhoff-Love displacement field [27]. That is, the components of the displacement field for sub-domain $i \in 0 \ldots N_{p}$ are:

$$
\begin{aligned}
u_{i \alpha}\left(x_{1}, x_{2}, x_{3}, t\right) & =-x_{3} w_{i, \alpha}\left(x_{1}, x_{2}, t\right) \text { for } \alpha=\{1,2\} \\
u_{i 3}\left(x_{1}, x_{2}, t\right) & =w_{i}\left(x_{1}, x_{2}, t\right)
\end{aligned}
$$

In this paper, partial spatial derivatives are denoted $\frac{\partial f\left(x_{1}, x_{2}, t\right)}{\partial x_{1}}=f_{, 1}\left(x_{1}, x_{2}, t\right)$, time derivative by dots e.g $\frac{\partial f\left(x_{1}, x_{2}, t\right)}{\partial t}=\dot{f}\left(x_{1}, x_{2}, t\right)$. Vectors are denoted in bold and $\mathbf{v}^{\top}$ is the transpose of a vector $\mathbf{v}$. Matrices are in bold between brackets.

In the bimorph regions, the bonding is supposed to be perfect, hence the piezoelectric ceramics and the substrate share the same displacement field. 

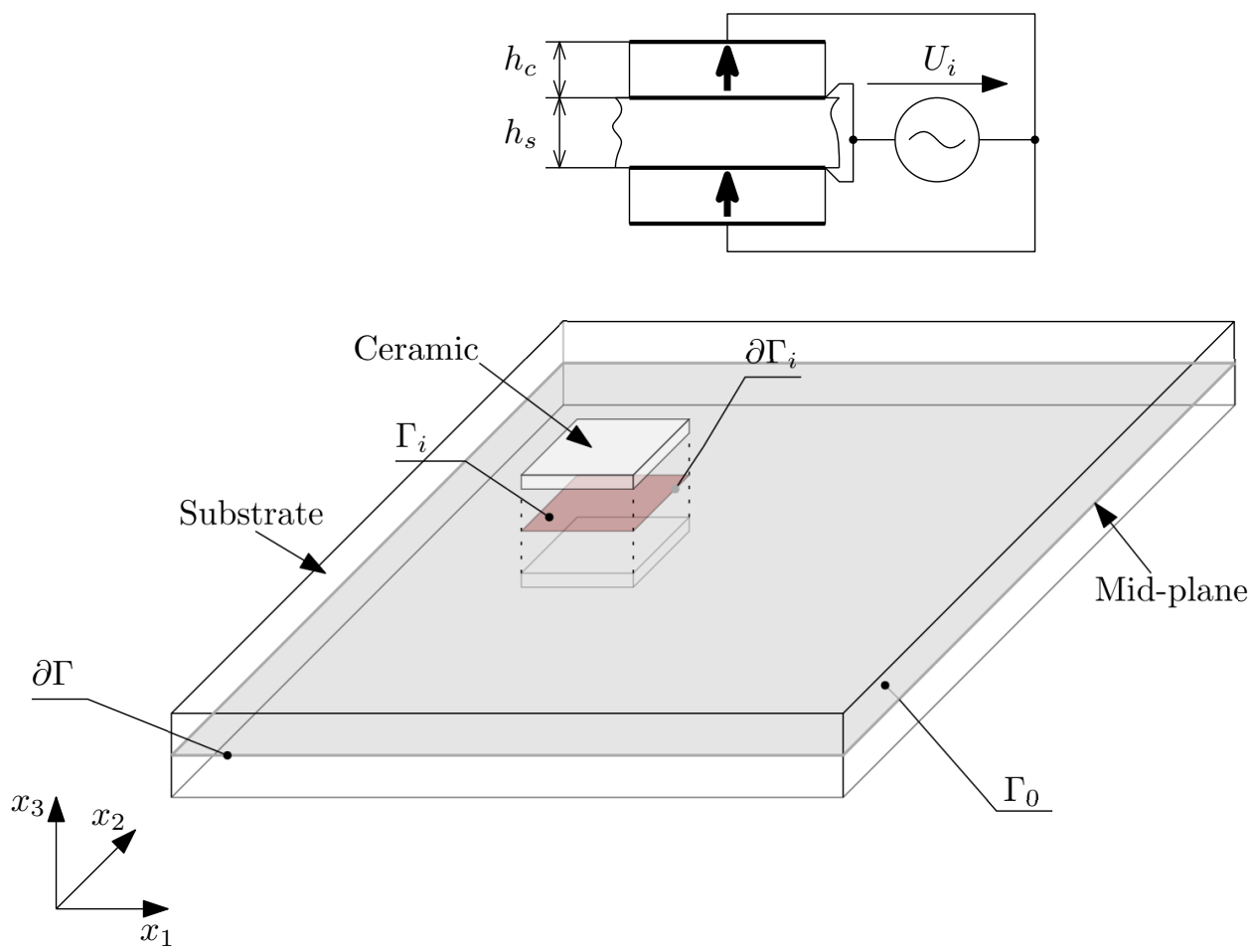

Figure 1: Schematic of the modelled device with the electric connexions of a ceramic pair (above) and the mechanic layout (below). Only one ceramic pair is represented for clarity. 
Using engineering notation, the non-nil components of the strain tensor for the sub-domain $i$ are:

$$
\begin{aligned}
S_{i 1} & =-x_{3} w_{i, 11} \\
S_{i 2} & =-x_{3} w_{i, 22} \quad i \in\left\{0 \ldots N_{b}\right\} \\
S_{i 6} & =-2 x_{3} w_{i, 12}
\end{aligned}
$$

The substrate is supposed to be linear hence the stresses are given by $\mathbf{T}_{s i}=[\mathbf{c}] \mathbf{S}_{i}$, where $[\mathbf{c}]$ is the stiffness tensor.

Considering now the piezoelectic ceramic, linear piezoelectric constitutive laws relating strain and stresses to the electric field $\boldsymbol{E}$ and the electric displacement vector $\boldsymbol{D}$ are used. Considering the electric enthalpy, they are given, for the considered subdomain $i \in\left\{1, \ldots, N_{b}\right\}$ by $[28,29,30]$ :

$$
\begin{aligned}
\mathbf{D}_{p i} & =[\mathbf{e}] \mathbf{S}_{i}+\left[\boldsymbol{\beta}^{S}\right] \mathbf{E}_{i} \\
\mathbf{T}_{p i} & =\left[\mathbf{c}^{E}\right] \mathbf{S}_{i}-[\boldsymbol{e}]^{\top} \mathbf{E}_{i}
\end{aligned}
$$

for the direct and converse effects respectively.

In practice, the piezoelectric patches used to exploit the transverse effect are thin and electroded on the upper and lower surfaces. Since the electric potential is usually prescribed on the electrodes, and assuming that thickness ratio between ceramics and substrate is small, it is commonly admitted that the electric field can be considered to be imposed by the voltage $U_{i}$ applied across the electrodes with a good approximation [31, 30]. Hence, it will be considered that $\left|E_{i 3}\right|=\frac{\left|U_{i}\right|}{h_{p}}$. As depicted in fig. 1, the polarizations of the patches and the voltage must be combined so that the piezoelectrically induced stress in each patch results in a moment.

Finally, the displacement field is continuous, that is the out of plane displacement and the rotation at a boundary between two sub-domains $i$ and $j$ should be equal that is:

$$
\begin{aligned}
w_{i} & =w_{j} \\
w_{i, \alpha} & =w_{j, \alpha} \quad \alpha \in\{1,2\}
\end{aligned}
$$

As for the excitation force, only the effects of the piezoelectric patches are included in the following. The excitation forces are supposed to be produced only by the piezoelectric patches.

\subsection{Equations of vibrations}

In the frame of the assumptions above, the equations of motion can be derived by applying Hamilton principle and are similar to the classical plate 
theory with segmented piezoelectric patches [32]. The dynamic equilibrium of a subdomain is given by :

$$
\mathcal{M}_{i} \ddot{w}_{i}+M_{\alpha \beta, \alpha \beta}^{i}=0 \quad \text { in } \quad \Omega_{i}
$$

where $\mathcal{M}_{i}$ the masses per surface unit and $M_{\alpha \beta, \alpha \beta}^{i}$ are the moment in the plate, which expressions are given in the appendices.

The overall displacement field is continuous, hence on a boundary $\partial \Gamma_{i}, i \geq 0$ that defines a bimorph domain, the following kinematic constraint is verified:

$$
\begin{array}{r}
w_{i}-w_{0}=0 \\
w_{i, n}-w_{0, n}=0
\end{array}
$$

$n$ indicating the normal direction of the local frame defined on the path $\Gamma_{i}$. To fulfil the equilibrium on the boundary the additional conditions must hold

$$
\begin{aligned}
M_{n n, n}^{0}+2 M_{n t, t}^{0} & =M_{n n, n}^{i}+2 M_{n t, t}^{i} \\
M_{n n}^{0} & =M_{n n}^{i}-M_{U_{i}}
\end{aligned}
$$

$t$ denoting the tangential direction of the local frame defined along the boundary. In Eq. 12, the moment $M_{U_{i}}$ is the moment produced by the ceramics under the voltage $U_{i}$, which results from the variational procedure [33]:

$$
M_{U_{i}}=\kappa_{b} U_{i} \text { with } \kappa_{b}=e_{31} d
$$

At the discontinuity points of $\Gamma_{i}$, the conditions (11) degenerate, yielding the Kirchhoff's corner load. Assuming that the material axes are parallel to the structural axes, this equivalent corner load is:

$$
M_{12}^{0}\left(\boldsymbol{x}_{c}\right)=M_{12}^{i}\left(\boldsymbol{x}_{c}\right)
$$

at the corners at coordinates $\boldsymbol{x}_{c}$. It can be noted that, as a result of the previous assumption, there is no contribution of the piezoelectric effect [34]. On the external boundary $\Gamma$, one has:

$$
\begin{array}{rll}
w_{0}=0 & \text { or } & M_{n n, n}^{0}+2 M_{n t, t}^{0}=0 \\
w_{0, n}=0 & \text { or } & M_{n n}^{0}=0
\end{array}
$$

and at the corners of $\Gamma$, the condition (14) becomes:

$$
M_{12}^{0}\left(\boldsymbol{x}_{c}\right)=0
$$




\subsection{Projection}

The modal analysis suppose to cancel the exogenous sources in the previous equation, hence $U_{i}=0$. This defines the Sturm-Liouville problem of the device. It turns out that the problem is merely a laminated and staged elastic plate for those conditions. It is well known that such problems have eigenvalues, the resonant frequencies $\omega_{k}$, and eigenvectors, the mode shapes that will be denoted $\varphi_{k}$.for the modal analysis the exogenous sources are cancelled, that is $U_{i}=0$. So the problem is merely a laminated and staged elastic plate, for which it is possible to find resonant frequencies $\omega_{k}$ and mode shapes denoted $\varphi_{k}$. If the latest are normalized according to the vibrating mass, they verify the orthogonality condition:

$$
\sum_{i=0}^{N} \int_{\Omega_{i}} \mathcal{M}_{i} \bar{\varphi}_{k} \bar{\varphi}_{l} d \Omega=\delta_{k l}
$$

where $\delta_{k l}$ is the Kronecker symbol, and $\bar{\varphi}_{k}$ denotes the normalized mode $k$. Let $M_{\alpha \beta}^{i k}$ be the moments for the modecalculated with the mode shape $\bar{\varphi}_{k}$ on the domain $\Gamma_{i}$, it follows from the previous property that

$$
\sum_{i=0}^{N} \int_{\Omega_{i}} M_{\alpha \beta}^{i k} \bar{\varphi}_{l, \alpha \beta} d \Omega=\delta_{k l} \omega_{k}^{2}
$$

The mode shapes $\bar{\varphi}_{k}$ constitute a basis, and therefore any shape $w\left(x_{1}, x_{2}, t\right)$ respecting the kinematic conditions on the boundaries can be decomposed as a weighted superimposition on the mode shapes i.e.

$$
w\left(x_{1}, x_{2}, t\right)=\sum_{k=1}^{\infty} \bar{\varphi}_{k}\left(x_{1}, x_{2}\right) \eta_{k}(t)
$$

where the modal coordinates $\eta_{k}$ depend solely on time. Projecting the equations (8) verified by $w$ as follows

$$
\sum_{i=0}^{N_{b}} \int_{\Omega_{i}}\left(\mathcal{M}_{i} \ddot{w}+M_{\alpha \beta, \alpha \beta}^{i}\right) \bar{\varphi}_{l} d \Omega=0
$$

Performing the integration by parts and using properties (18), (19), results in a set of decoupled dynamic equations that govern the modal coordinates

$$
\ddot{\eta}_{k}+\omega_{k}^{2} \eta_{k}=\sum_{i=1}^{N_{b}} \oint_{\partial \Gamma_{i}} M_{U_{i}} \bar{\varphi}_{k, n} d \Gamma
$$




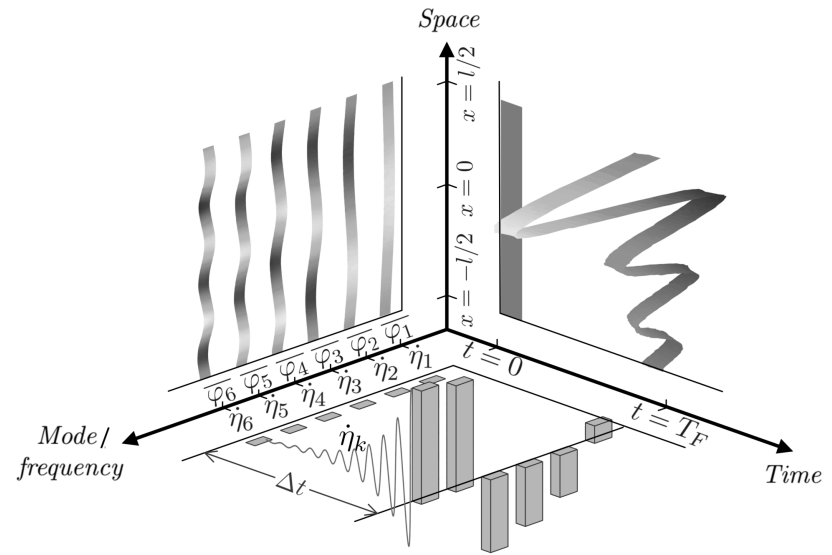

Figure 2: Schematic of the method: the projections of the desired velocity field yield the final modal coordinates at focusing time in the modal space, their evolutions must then be specified by individual time functions between the rest and the focus configurations

where the contribution of the piezoelectric patches to the mode appears as the work produced by the piezoelectric moment for the rotation around the tangential axis along the periphery of the ceramic. It follows from this equation that several voltages applied to several ceramics can participate to the excitation of a given mode, and reciprocally, that a ceramic can contribute to excite several modes.Indeed, since $U_{i}$ is constant in a ceramicthe contribution to the modal force exciting a mode $k$ is

$$
\oint_{\partial \Gamma_{i}} M_{U_{i}} \bar{\varphi}_{k, n} d \Gamma=\kappa_{b} \oint_{\partial \Gamma_{i}} \bar{\varphi}_{k, n} d \Gamma U_{i}=\alpha_{i k} U_{i}
$$

where $\alpha_{i k}$, the participation coefficient of a ceramic $i$ to a mode $k$ per unit voltage, is fixed by the design, in other words the location, the dimensions - and ideally the shape - of the patches.

\subsection{Strategy}

The two results given by (20) and (23) are now exploited to specify a velocity field and a transient. The problem can be stated as sketched in fig. 2. The first step is to define the modal coordinates. Therefore, given a reference velocity field $\dot{w}^{\star}$ that should be realized at a given focusing time $t_{f}$, we first compute its velocities spatial spectrum by:

$$
\dot{\eta}_{k}^{\star}=\sum_{i=0}^{N} \int_{\Omega_{i}} \mathcal{M}_{i} \bar{\varphi}_{k} \dot{w}^{\star} d \Omega
$$


This specifies only the spectrum to be realized at the specified focusing time $t_{f}$, that is $\dot{\eta}_{k}\left(t_{f}\right)=\dot{\eta}_{k}^{\star}$, but the time evolution is still to be defined. Arbitrarily setting the transients for each modal coordinates is however difficult as it would probably result in unrealistic dynamic involving very high voltages. In this paper, we adopt a different strategy based on the following observations:

1. Exciting an oscillator at its resonance frequency requires a minimal excitation force;

2. Rewriting the eq. (22) as a function of the modal velocities coordinates and taking the Laplace transform gives

$$
\mathcal{L}\left[\dot{\eta}_{k}\right](s)=\frac{s}{s^{2}+\omega_{k}^{2}} \mathcal{L}\left[\psi_{k}\right](s)
$$

where $\mathcal{L}$ is the Laplace transform with respect to time, and $s$ the Laplace variable and the modal force $\psi_{k}$ is defined according to eq. 22

$$
\psi_{k}=\sum_{i=1}^{N_{b}} \alpha_{i k} U_{i}
$$

This last equation shows that the modal velocity of a mode $k$ results from applying a band-pass filter to the modal force that is produced from a linear combination of voltages. Therefore, it is to possible to selectively excite a mode by using a time-varying modal force such that its spectrum includes the relevent frequency $\omega_{k}$. For this approach to be valid however, the modes should not be too close in the frequency domain or coupled because of the damping. Hence, the following discussion holds for weakly damped structures.

To exploit these consideration, a modal force is now researched as a function modulated by an harmonic function at $\omega_{k}$ as sketched in fig. 2. The purpose is to impose the envelop of the excitation of each mode independently to gain some control on their transient. Supposing that the frequency decoupling assumption holds, consider the modal dynamic equation where the damping $\xi_{k}$ of the mode is now introduced:

$$
\ddot{\eta}_{k}+2 \xi_{k} \omega_{k} \dot{\eta}_{k}+\omega_{k}^{2} \eta_{k}=\psi_{k}
$$

and suppose, as an illustration, that the desired time evolution of the modal velocity is

$$
\dot{\eta}_{k}(t)=A_{k} \mathrm{e}^{\gamma_{k} a_{k} t} \sin \left(b_{k} t\right)
$$


where $a_{k}=\xi_{k} \omega_{k}, b_{k}=\sqrt{1-\xi_{k}^{2}} \omega_{k}$ are the damping factor and the natural frequency of the mode, $A_{k}$ and $\gamma_{k} \geq 0$ are some factor that scales the transient. Using the Laplace transform for instance, one calculates the following expression for the modal force:

$$
\psi_{k}(t)=\Psi_{k} \mathrm{e}^{\gamma_{k} \xi_{k} \omega_{k} t}\left[B_{k} \sin \left(b_{k} t\right)+C_{k} \cos \left(b_{k} t\right)\right]+D_{k}
$$

The details of the various constants $B_{k}, C_{k}$ and $D_{k}$ can be found in the appendices.

To define the transient, $\gamma_{k}$ and $A_{k}$ still have to be fixed. This is done in this work by setting the time $\Delta t_{k}=t_{f}-t_{k}$, where $t_{k}$ is the starting time of the transient, allocated to each mode to reach its final value. It is also imposed that $\omega_{k} \Delta t_{k}=\frac{\pi}{2}+2 \pi N_{k}\left(N_{k} \in \mathbb{N}\right)$. This ensures that all the modal coordinates reach their maximum value at the end of the focalisation (this constraint is actually a simple way to ensure that if only one ceramic is used, the maximum voltage is easily handled). The parameter $\gamma_{k}$ is used to ensure that the transient is below some level during a given time in view of the haptic interaction. Finally $A_{k}$ is calculated by:

$$
A_{k}=\frac{\eta_{k}^{\star}}{\exp \left(\gamma_{k} a_{k} \Delta t_{k}\right)}
$$

In fig. 3 , the case $N_{1}=6$ for $\gamma_{k} \in\{1,10,50\}$ is presented in a simulation (unit are normalized with regard to the mass i.e speed are in $\mathrm{kg}^{-0.5} \mathrm{~mm} \mathrm{~s}^{-1}$ and force in $\mathrm{kg}^{-1 / 2} \mathrm{~N}$. The dynamic model is constituted by two modes having the same modal damping $\xi_{1}=\xi_{2}=0.01$ and such that $\omega_{2}=2 \omega_{1}$. The simulation is ran applying $\psi_{1}$ to the first mode. The velocities for each modes and the input are depicted. It can be noted that the first mode is indeed controlled (fig. 3-a) in every case. In this example, for $\gamma_{1}=1$, the offset $D_{1}$ is large (fig. 3 -c), and produces an undesirable transient effect on the second mode (fig. 3-b), which would be a problem if both modes where to be controlled simultaneously. For $\gamma_{1}=10$, the transient is modified in a such a way that the amplitude remains below half the amplitude at focusing time for nearly $95 \%$, with limited effect on $\varphi_{2}$. Finally, if the first mode is too strongly boosted, the input is very rapidly increasing at the end of the transient, meaning that its spectrum is spread. This induces a "spill-over" of the excitation that now also energize the second mode. Therefore, caution is required when accelerating a mode for this reason, but also because the modal force -that is the voltage required-increases. Indeed, practically, the voltage applied should not exceed some limits. One possibility is to take 

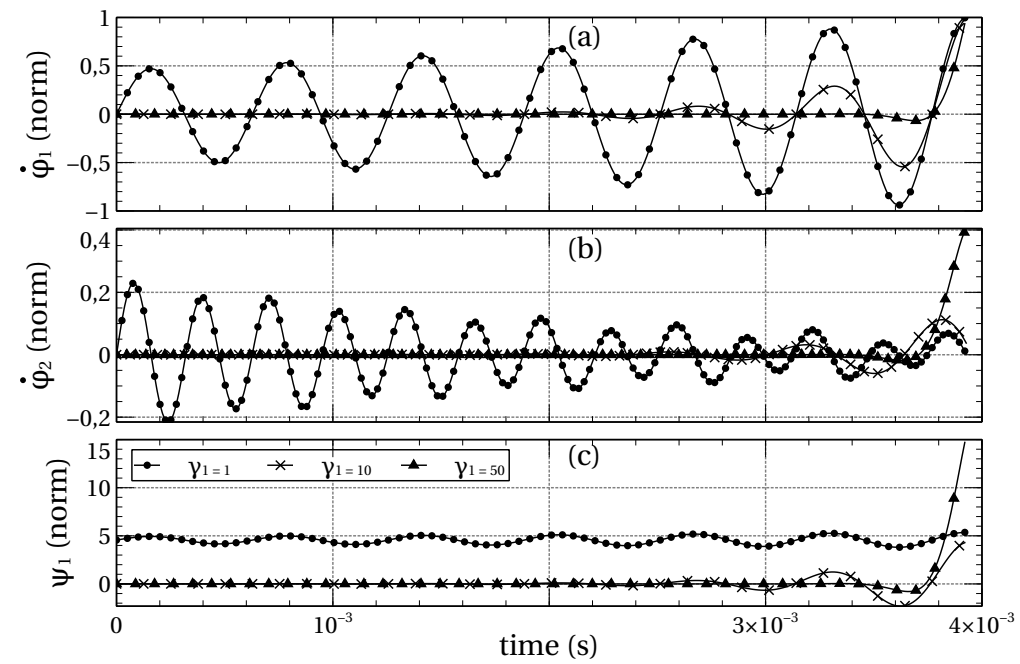

Figure 3: Example of transient in the case where mode $\varphi_{1}$ (a) is excited by the modal force (c) to impose an transient which growth is controled by the factor $\gamma$. The neighboring mode $\varphi_{2}$ (b) can be excited either by either by the offset (for $\gamma=1$ or too fast a transient $\gamma=50$.

advantage of the multiplicity of the actuators to distribute the voltages as evenly as possible among them. To do so, the voltages $U_{i}$ are rewritten as a linear combination of the modal forces calculated above:

$$
U_{i}(t)=\sum_{k=1}^{n_{m}} m_{i k} \psi_{k}(t)
$$

where the weights $m_{n k}$ are used to allocate the desired modal force among the ceramics. Thus, the modal dynamic equation is now:

$$
\ddot{\eta}_{k}+\omega_{k}^{2} \eta_{k}=\sum_{i=1}^{N_{b}} \alpha_{k i}\left(\sum_{k=1}^{n_{m}} m_{i k} \psi_{k}(t)\right)
$$

Assuming that the modes can be excited separately owing to the frequency selectivity, we require the conditions:

$$
\sum_{i=1}^{N_{b}} \alpha_{k i} m_{i k}=1 \text { for } k=\left\{1, \ldots, n_{m}\right\}
$$

to ensure that the necessary modal force is are indeed applied. Then, the weight can be tuned to reduce the voltages $U_{i}$ actually applied to the ce- 
ramics by stating the following optimization problem:

$$
\begin{array}{ll}
\underset{\boldsymbol{m}_{\boldsymbol{i}}}{\operatorname{minimize}} & \|\boldsymbol{M} \boldsymbol{\Psi}\| \\
\text { subject to } & \left|U_{i}\right| \leq U_{\max }, i=1, \ldots, N_{b} . \\
\text { and to } & \sum_{i=1}^{N_{b}} \alpha_{k i} m_{i k}=1 \text { for } k=\left\{1, \ldots, N_{m}\right\}
\end{array}
$$

where $\boldsymbol{M}$ is the matrix of the weights (which coefficients are the $m_{i k}$, $\left.\operatorname{dim}(\boldsymbol{M})=N_{b} \times N_{m}\right)$. $\Psi$ is a matrix which $k$-th row vector is constituted by ordered samples of the modal forces $\psi_{k}\left(n T_{s}+t_{0}\right)$. The number of samples $N_{s}$ and the sampling should be chosen such that $N_{s} T_{s}+t_{0}=t_{f}$, and to cover a time interval where the modal forces $\psi_{k}$ become large with respect to the voltages rating. In this work, the Frobenius norm was used.

The results in fig. 4 illustrate the procedure. In this simulation, the voltages required with and without optimization are compared in fig. 4-a, together with the related transient are presented fig. 4-b. The same system as the previous simulation is considered, but this time, to model two ceramics, different modal piezoelectric coefficients are considered. In this scenario, the voltage limitation is set to $20 \mathrm{~V}$, the two modes dynamics are accelerated by choosing $\gamma_{1}=4$ and $\gamma_{2}=2$. It is also required that $N_{1}=10$ and $N_{2}=15$, which results in a large offset for the first mode especially (dashed- black circles curve), the second mode (dashed-white diamonds) is less impacted because of the larger number of cycles allowed. The optimisation is performed considering the two last oscillations of the first mode (which exceed $40 \mathrm{~V}$ peak to peak). The simulation shows that the procedure indeed imposes new voltages that respect the given limitation almost everywhere during the transient. The transient (bottom) are quasi identical as expected.

\section{Experimental results}

\subsection{Set-up}

The experimental set-up is depicted in fig. 5. The device is fixed on a $\mathrm{x}-\mathrm{y}$ table in order to position it under the vibrometer laser head (OFV 505 Polytec) to measure the vibration velocity at various locations. All measurements are monitored and recorded using an oscilloscope (picoscope 3403D pico technolgies LTD). The voltages are pre-calculated using the procedure described earlier, and sent to a two channel arbitrary waveform generator (Tektronix AFG3022B). These voltage references are then amplified thanks to two analog amplifiers supplying bipolar voltages up to $150 \mathrm{~V}$ (NF HSA 


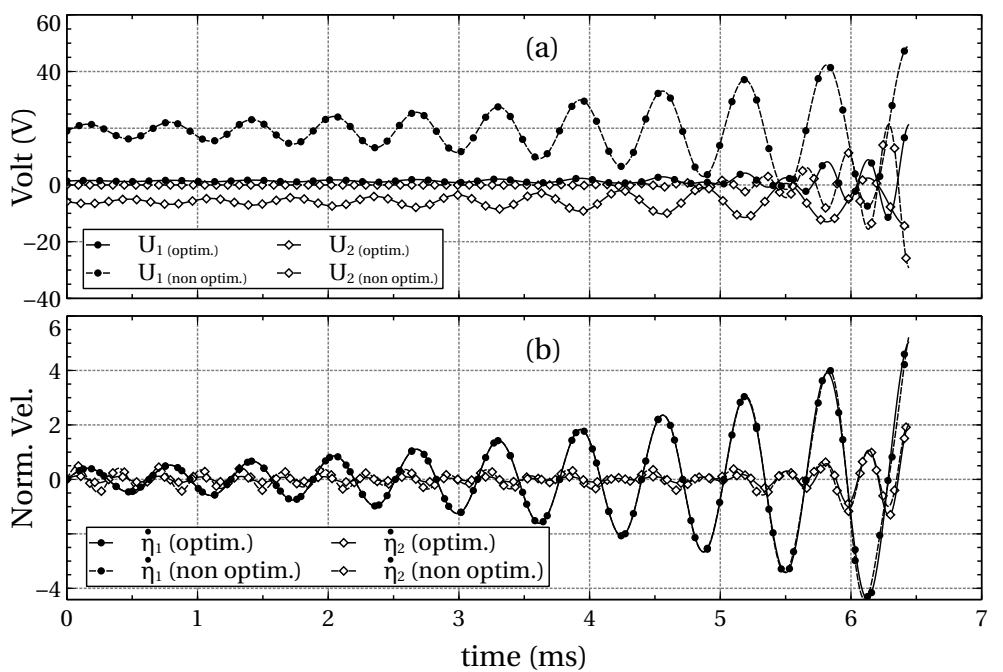

Figure 4: Effect of allocating the modal forces among two ceramics. Voltages $U_{1}$ and $U_{2}$ (top) needed in the case of assignement of one ceramic to excite one mode are in dashed, and in plain for the case of optimized allocation. The transient are reaching the desired modal speed in both case (bottom).

4051). Since the phenomenon under investigation is essentially a transient, measurements and waveform generation are synchronized using an external synchronization clock. It generates a square waveform at a low frequency that is chosen to let the whole process of focalisation then decaying happen, in order to repeat the measurements starting from the same initial condition. The waveforms are acquired using the same signal and to ensure that the lag between the trigger signal and the actual generation is known, the clock signal was also measured. In practice, this lag is negligible. The voltages featuring multiple frequencies up to $100 \mathrm{kHz}$, it is required to respect the Shannon frequency. In the case of the generator used, for a focalisation time of $50 \mathrm{~ms}$, the equivalent sampling frequency with the $2^{16}$ points available is $1.3 \mathrm{MHz}$ which verifyies the criterion. The waveforms specification and the measurements processing are performed by homemade software using matlab and the libraries provided by pico technology and tektronix.

The demonstrator is a clamped staple shaped aluminium beam equipped with piezoelectric patches Noliac NC51 with dimensions $(9 \times 5 \times 0.5) \mathrm{mm}^{3}$ as visible on the picture in fig. 6. This peculiar shape was chosen because i) it reduces the surface to be scanned hence the velocities cartography are considerably faster to realize; ii) it demonstrates that the method is usable 


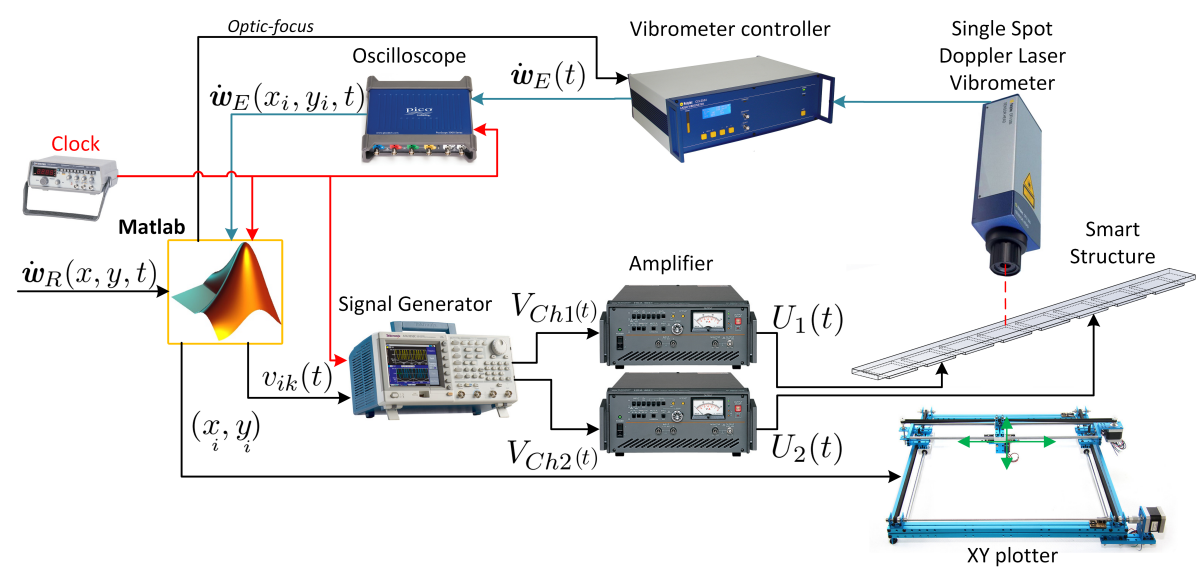

Figure 5: Schematic of experimental setup.

even if actual solutions to the eigen problem are not known. As discussed above, the ability of a voltage $U_{i}$ to excite the $k$-th mode depends on the $\alpha_{i k}$ coefficient eq (22) which depends on the geometry and location of the patchess. To avoid to use different ceramics, the electrodes were actually splitted (e.g second ceramic from the left). By properly chosing these cuts, it is possible to evenly excite the modes.

The identification was realized using a white noise identification procedure. The velocity vs input voltage frequency response fonction was recorded at points defined on a regular grid on the upper part of the beam. The mapped region was defined between the limits of the curved parts, with a step small enough with regard to the smallest spatial wavelength. During the process the end ceramics were used in conjunction or in opposition, to excite the even and odd modes respectively, because they are able to excite all the bending modes in the $100 \mathrm{~Hz}-80 \mathrm{kHz}$ band. During these measurements, the other ceramics are in short-circuit conditions. Modes were selected using the resonance peaks. Knowing the phase and amplitude at resonant frequencies at each measured point, the mode shapes were reconstructed. When the signal to noise ratio was too low, the mode shapes were more precisely mapped at resonance choosing ceramics with more effective participation coefficients.

In fig. 7 , the modes $\varphi_{1}$ to $\varphi_{15}$ (up to $30.9 \mathrm{kHz}$ ) are depicted in grey. The orthogonality of the measured modes, however, is only approximatively verified, as shown in fig. 8 on the left, where the result of the normalized projection of modes on each other reveals cross talk (e.g between $16^{\text {th }}$ and 


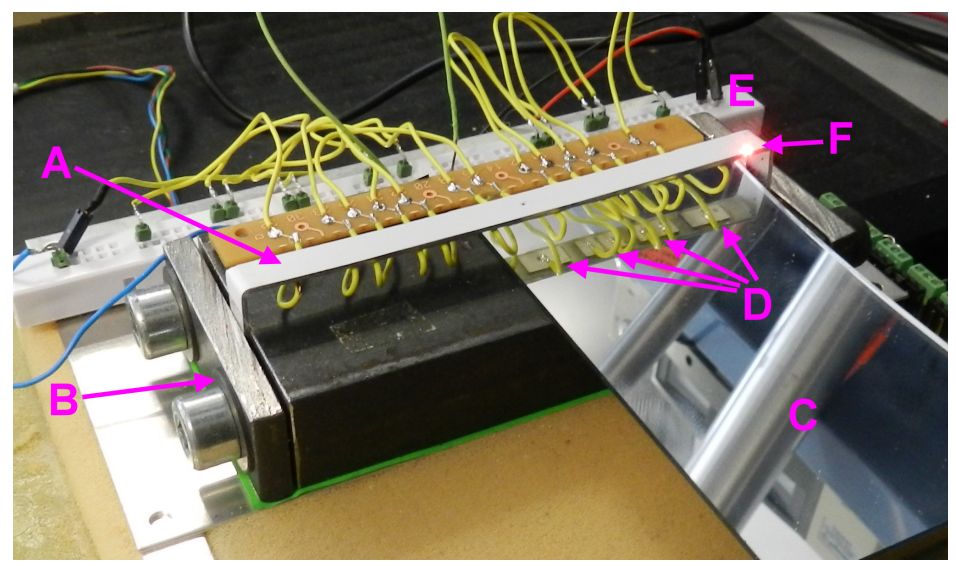

Figure 6: Picture of the experiment: A) Staple shaped aluminum beam, the velocity is controlled within a rectangular region on the upper part; B) Fixture of the beam to realize clamp-clamp boundary conditions; C) Mirror showing the ceramics; D) Reflection revealing some of the ceramics fixed beneath the beam. The second ceramic from the left is split as explained in the text; E) Electrical connections of transducers allowing the reconfiguration according to the set of ceramics used; F) Laser spot positioned at one of the corner of the studied region.

$17^{\text {th }}$ mode). This results from the mapping that does not cover the whole device, but only a rectangular area on the top. Indeed, finite element simulation showed that for low frequency modes (having large wavelength) the corners of the staple where acting as clamps, i.e. the modes were located on the top of the staple only. However, as the frequency increases, this is not true any more, and modes tend to occupy the whole staple. This is also verified experimentally. To address this problem, the modes are orthogonalized using a Gram-Schmidt procedure which gave significant improvement (fig. 8 right) although the shapes are only slightly modified (depicted in color in fig. 7). The time synthesis of the voltages discussed previously requires a fair knowledge of the damping of a mode, which was done by measuring the $-3 \mathrm{~dB}$ bandwith. Finally, the participation factors were estimated from the measurement of the velocity at resonance obtained when supplying each ceramic individually (the other being short-circuited).

\subsection{Protocol}

The main experiments presented here aimed at testing the viability of the approach to reproduce a specified shape on the top of the "staple". A sinc function, where position, width and amplitude of a relatively homogeneous high velocity region can be easily specified, was adopted. From fig. 7, 

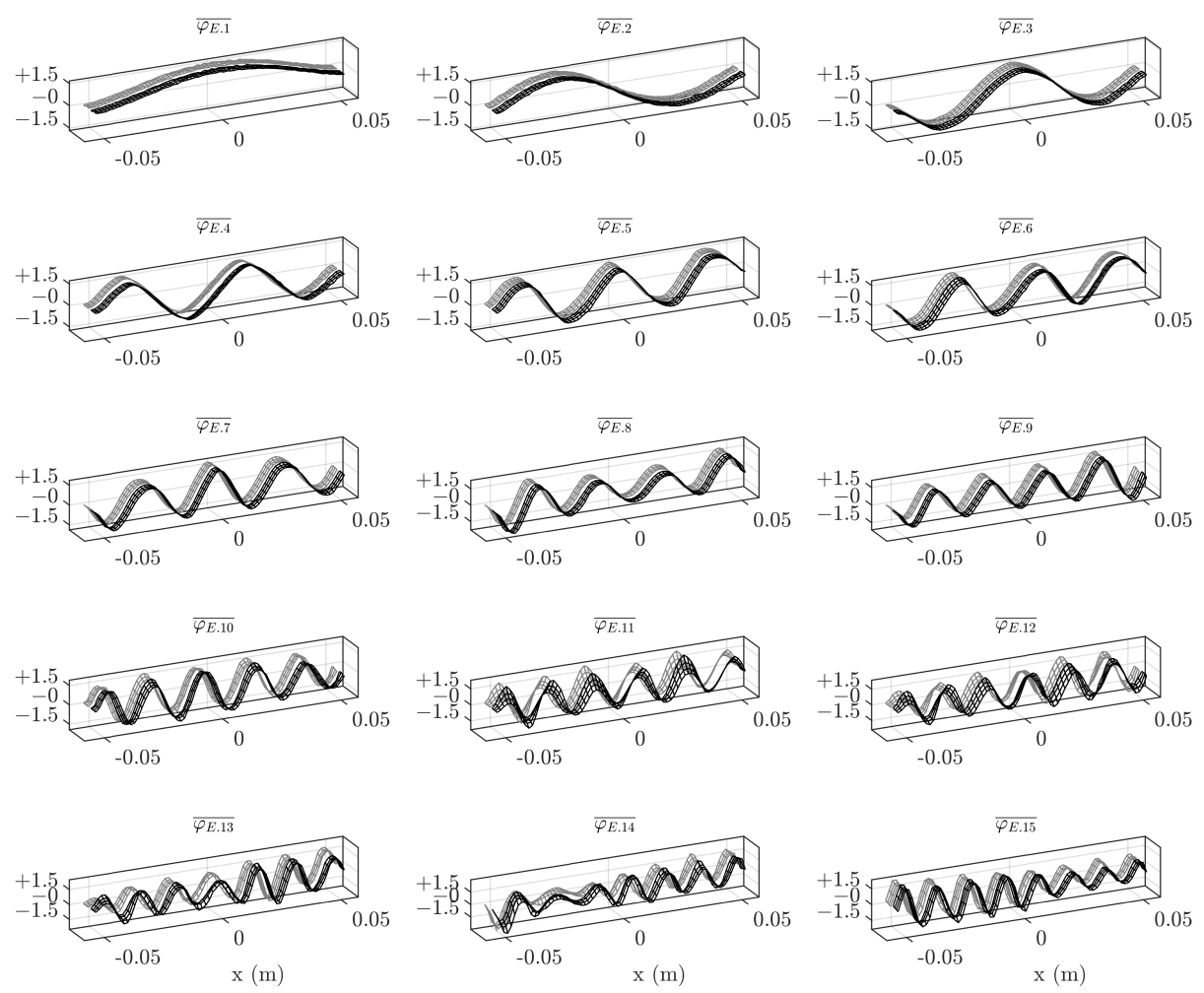

Figure 7: Experimental mode shapes (grey) and reorthogonalized mode shapes (colored) in the frequency range 0 to $39 \mathrm{kHz}$ 

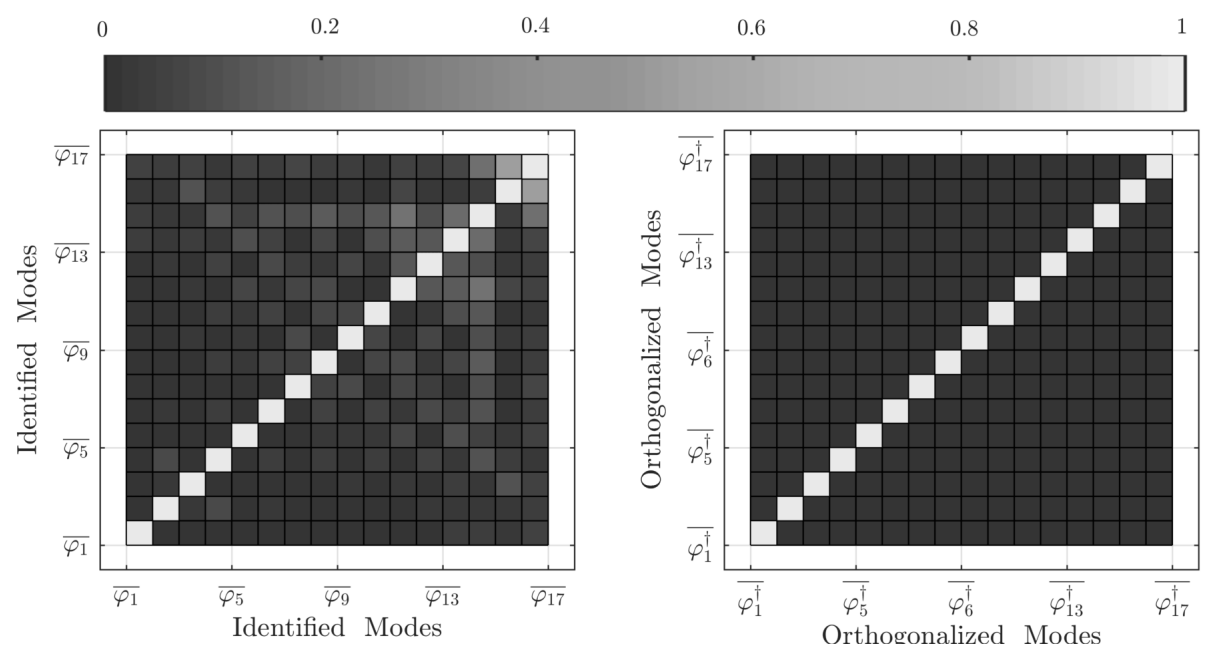

Figure 8: Cross projection between experimental modes reveals their imperfect orthogonality (left) which can be rectified using an orthogonalization procedure (right)

one can observe that the first modes are relatively similar to harmonic functions. The clamp-clamp modal shapes of a beam can be well approximated by cosine function from the third mode. To understand the trend of the relationship between mode amplitude and the reference shape, we assimilate all the modal shape to cosine function $\varphi_{k}(x)=\cos \left(\beta_{k} x\right)$ for $\beta_{k}=\frac{k \pi}{L}$ ( $L$ :length of the beam). Here, without loss of generality, the normalization factor is omitted as it is the unity for all modes of a uniform beam . This approximation leads to overestimate the contribution of the first two modes, but provides insight of the general trends found later. Under those assumptions, the projection of the reference function is

$$
\int_{-0.5}^{0.5} \operatorname{sinc}(a x) \cos \left(\beta_{k} x\right) d x=\frac{\operatorname{Si}\left[\frac{1}{2}(k \pi-a)\right]+\operatorname{Si}\left[\frac{1}{2}(k \pi+a)\right]}{a}
$$

where $\mathrm{Si}$ is the integral sine. Hence a narrow "high velocity" region will require more modes for a satisfactory approximation. On the other hand, their amplitude decreases so it should be possible to realize velocity fields with "sharp" variation despite the voltage limitation. Actually, the modal spectrum follows the same trend as the Fourier transform of sinc function. Five different focusing locations, equidistantly distributed on the beam, were tested. For the outermost points and large central lobe of the sinc function, the kinematic condition cannot be respected. So a Tukey window is used to respected the boundaries of the cartographies. 
To assess the control of the mid height width of the central lobe, successive widths of 25, 18 and $10 \mathrm{~mm}$ were tested. Amplitude control was indirectly assessed by imposing $40 \mathrm{~mm} \mathrm{~s}^{-1}$ as the maximal velocity for every test (before applying the Kaiser window). For practical reason, the modal projection must be truncated. To select the modes, a criterion is defined as follow. Considering that twice the kinetic energy is given by:

$$
\mathcal{K}=\int_{\Omega} \rho \dot{\boldsymbol{w}}^{\top} \dot{\boldsymbol{w}} d \Omega=\int_{\Omega} \rho \overline{\boldsymbol{\varphi}}^{\top} \overline{\boldsymbol{\varphi}} d \Omega \dot{\eta}_{k}^{2}=\sum_{k=1}^{\infty} \dot{\eta}_{k}^{2}
$$

one can define the $\tau$-score criterion for a truncation order $N_{m}$ :

$$
\tau=\left(1-\sum_{k=1}^{N_{m}} \frac{\dot{\eta}_{k}^{2}}{\mathcal{K}^{2}}\right)^{\frac{1}{2}}
$$

where $\mathcal{K}$ can be determined directly from the specified velocity field $\dot{w}$. The $\tau$-score is thus a measure of the residue resulting from the truncation.

For each test, the velocity field was measured, projected on the modal basis and compared to the specified modal coordinates resulting from the truncation. The criterion $v$-score used to assess the "misalignement" of the obtained shape compared to the specified one is defined as:

$$
v=\frac{\operatorname{cov}\left[\dot{\boldsymbol{w}}^{\star}\left(x_{m}\right), \dot{\boldsymbol{w}}\left(x_{m}, t_{f}\right)\right]}{\sqrt{\operatorname{var}\left[\dot{\boldsymbol{w}}^{\star}\left(x_{m}\right)\right] \times \operatorname{var}\left[\dot{\boldsymbol{w}}\left(x_{m}, t_{f}\right)\right]}}
$$

where var and cov are the variance and the covariance respectively, and $x_{m}$ are the location where the velocities are sampled. The criterion is chosen to evaluate the discrepencies between the truncated modal projection and the experimental modal projection, regardless of the error on the amplitude which can be due to a miscalibration of the amplification gain. In this way, one can appreciate whether the obtained shape is similar to the (truncated) reference up to a scaling factor.

\subsection{Results}

\subsubsection{Voltages}

The strategy proposed to comopute the voltages applied is examined in this section. As explained in section 2.4, the voltages deduced from the prescribed modal forces given by eq. (26) can be "mixed" between the patches using a linear combination. Here, the scenario examined is to control two modes simultaneously, and force them to achieve a given set of velocities 


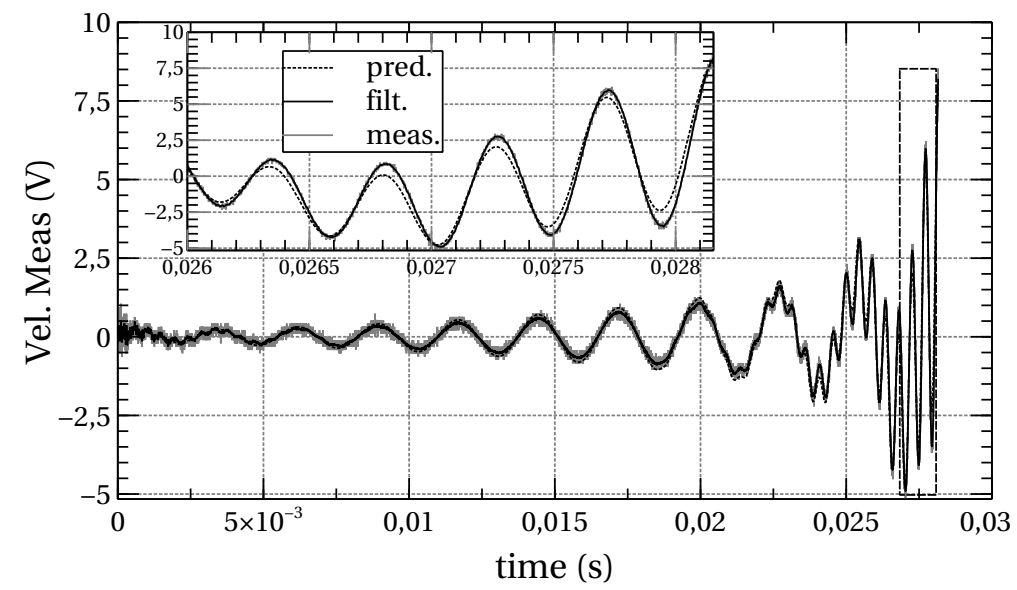

Figure 9: Comparison between predicted and measured velocities at the center of the staple: measurements (grey), filtered measurements (plain line, black) and predicted (dashed line, black). The gain of the velocity measurement is $5 \mathrm{~V} \mathrm{~s} \mathrm{~mm}^{-1}$. Zoom at focus time is depicted in the frame.

of $20 \mathrm{~mm} \mathrm{~s}^{-1}$ for the modes $\varphi_{1}$ and $\varphi_{3}$ at the center of the experimental setup (for simplicity, the modes are renormalize in order to have $1 \mathrm{~mm} \mathrm{~s}^{-1}$ amplitude for the maximum velocity of a given mode shape). Therefore, the expected velocity at the center is $40 \mathrm{~mm} \mathrm{~s}^{-1}$. Moreover, a speed up of $\gamma_{1}=4$ and $\gamma_{3}=1$ over the natural decaying speed of modes $\varphi_{1}$ and $\varphi_{3}$ is imposed. Finally, $\varphi_{1}$ and $\varphi_{3}$ should achieve the focusing within $N_{1}=10$ and $N_{3}=25$ oscillations respectively. For the purpose of the demonstration, two set of patches were selected. The first one (denoted set 1 in the sequel) has a strong effect on $\varphi_{1}$, but a very weak effect on the second, while the second set is more balanced with a prominent effect on $\varphi_{3}$. This results in the voltages depicted in fig. 10 (bottom).

In fig. 9, a comparison of the simulated and measured velocity transients is presented. The agreement is good despite the uncertainties on the dynamic parameters. To assess the effect of "mixing" the voltages, a second run consisted in applying the optimisation procedure in order to conceal the voltages amplitude below $50 \mathrm{~V}$ as long as possible during the transient. The resulting voltages (named case 2), depicted in fig. 10 (middle graph), are indeed respecting the constraint as far as possible. Due to the poor ability of patch 1 to energize the second mode, the optimization procedure favors the use of the second patch to reduce the voltage amplitudes. This is clearly visible on the spectra of fig. 11. This figure also confirms that the bandwidth 


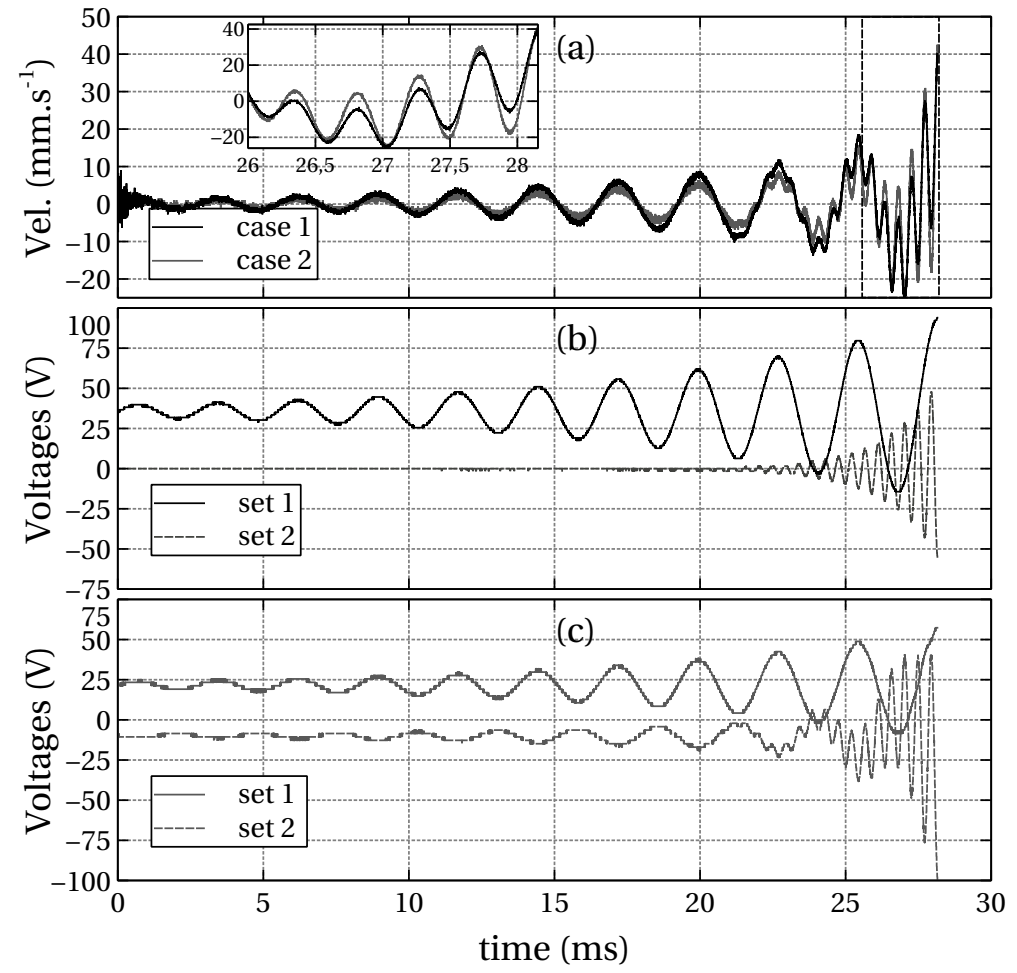

Figure 10: Validation of the voltages allocation strategy: (a) velocity measurement with "one mode one patch" strategy (case 1 black) and mixed voltages between patches (case 2 dark grey). The corresponding voltages applied to the patches set are depicted in the case 1 (b) and case 2 (c).

of the excitation signal is well controled since the second, 4 th and 5 th modes (at $f=963,3907$ and $5719 \mathrm{~Hz}$ )are not excited. The transients are still very similar as expected, and the velocity at focusing time is still realized.

\subsubsection{Location}

The results from tests to control the location at $x=-46,-24,0,24$ and $46 \mathrm{~mm}$ of the high velocity zone $(25 \mathrm{~mm}$ of width at mid-height of the main lobe) are presented in fig. 12. For each test, the specified, the approached and the measured velocities at focusing time along the center line in the y direction are presented (left column). The approached reference velocity field results from the truncation of the actual modal decomposition of the reference which was fixed here to 6 modes for all references in this case. As explained earlier, the reference field is windowed with a Tukey window 


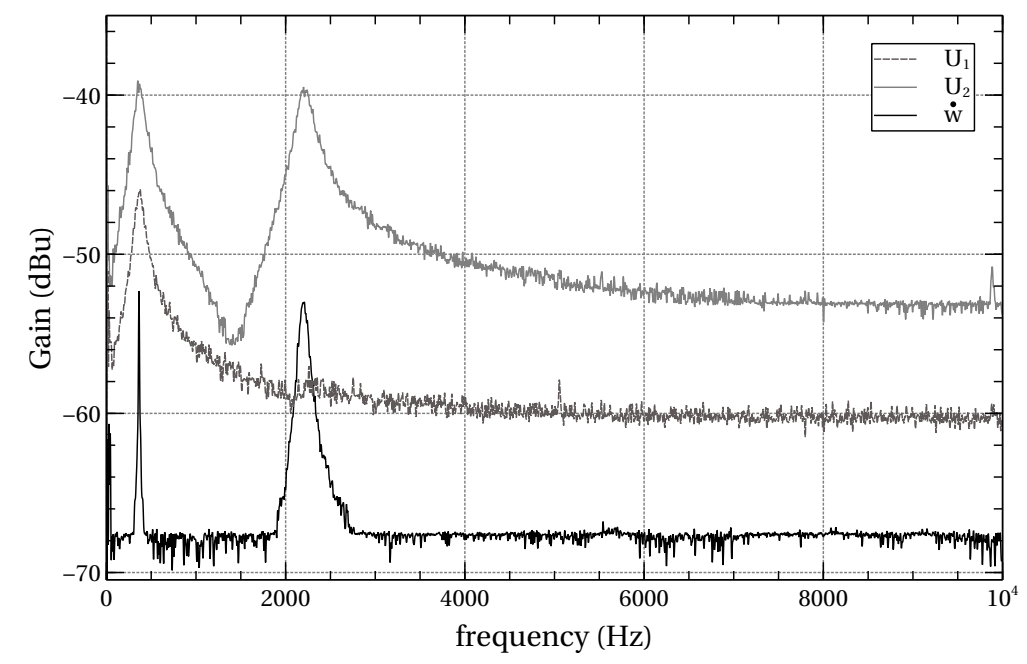

Figure 11: Spectra of the voltages and velocity at the center for case 2: since set 1 of ceramics is poorly coupled to the 3rd mode, the optimization requires that the set 2 is used also to excite mode 1 , hence reducing the voltages as shown in fig. 10

having a sharp transition to ensure a nil value at the boundary, without causing too much distortion of the initial reference.

Similar test where ran for sharper pulse. The $\tau-$ score of the various truncation are summarized in Tab. C.1 in the appendix Appendix C. One can observe that the higher the truncation the better the approximation of the velocity field, the worst cases being on the ends. This is due to the need to "cut-off" the actual waveform, which, even with the use of the Tukey-window, requires a wider spatial spectrum. This is clear from the improvement of the score with the reduction of the width of the pulse.

For each test, there is a good agreement of the approached and the measured velocity field, despite the imperfect knowledge of the damping and the participation factors. At the ends of the beam, it would require more modes to achieve the stiff edge of the reference. Hence, the assigned maximum velocity cannot be achieved, still the selected truncation order is high enough to achieve a correct reconstruction (see Tab. C.2).

\subsubsection{Width}

The results are summed up in fig. 13, with the same convention as before. As already explained, reducing the width of the main lobe of the sinc function, results in a smaller and wider spectrum in the modal basis and is therefore more challenging. One can observe on the spectrum that 

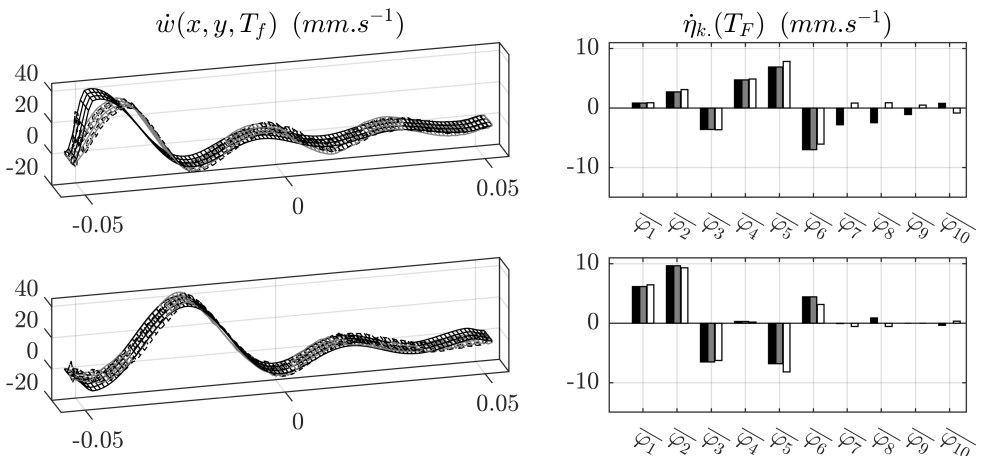

$\phi_{1} \phi_{2} \phi_{3} \phi_{7} \phi_{5} \phi_{6} \phi_{7} \phi_{8} \phi_{9} \phi_{10}$
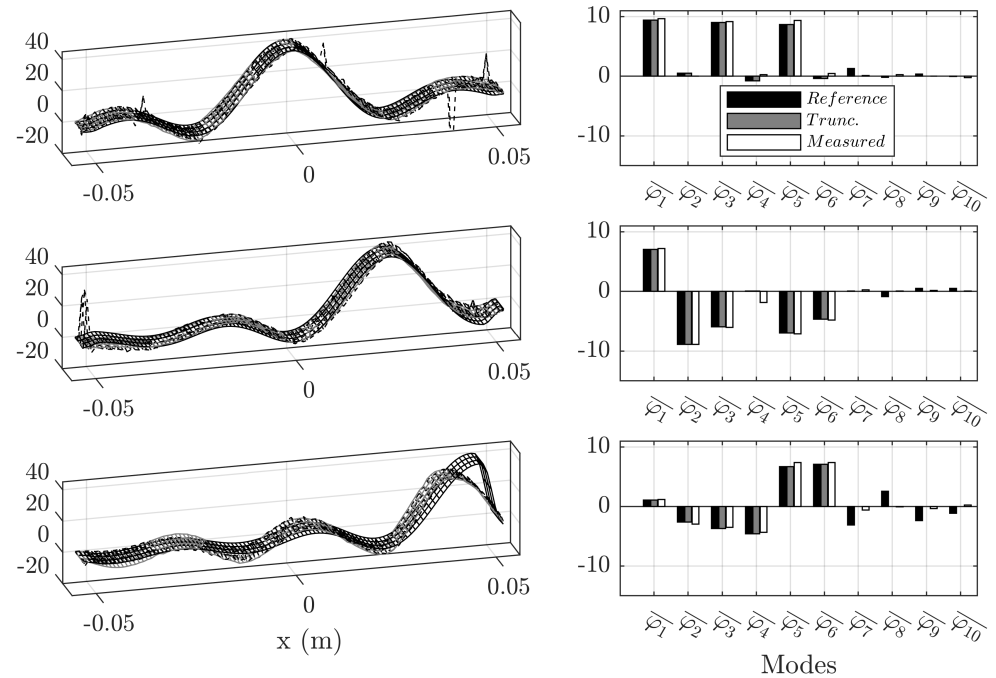

Figure 12: location test at $-46,-24,0,24$ and $46 \mathrm{~mm}$, on the left velocity fields for the references, the approached ones resulting from the truncation and the measured velocities (resp. black, grey and white) and their respective modal projection on the right. Note that for the ends velocity fields a Tukey window is applied to respect the kinematic boundary conditions. 

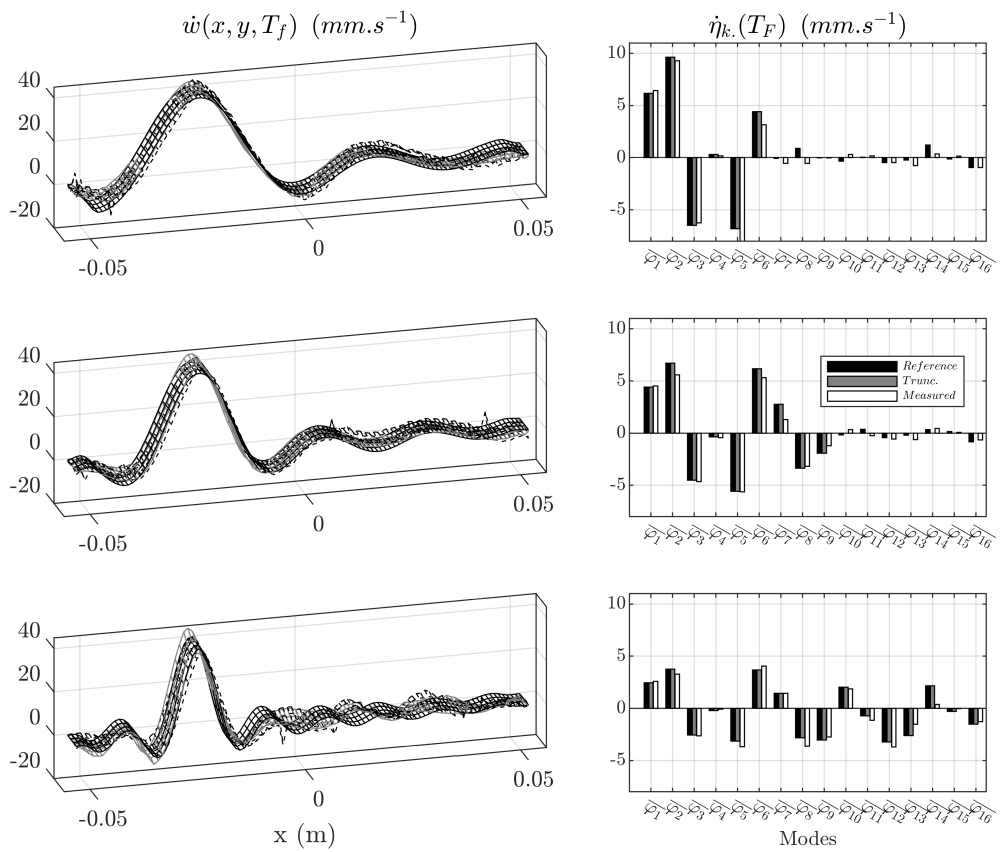

Figure 13: Width test results with the reference (black), the truncated reference (grey) and measured (white), the velocities and their modal projection are depicted in the left and right columns respectively.

higher order modes are not correctly excited. This is partly explained by the high speed transient of these modes: the pre-calculated signals also include the lower frequency transient which are much slower, hence a long signal (compared to the actual transient time of the high frequency modes) is needed. This results in a coarse sampling given the limited number of points that can be programmed into the generators. However the effect is not too critical on the actual velocity field.

\subsubsection{Transient}

The transient can be independently controlled by imposing the transient of the voltage harmonics. In fig. 14, the transients of the five first modes are depicted in the case where $\varphi_{1}$ is strongly accelerated. These measurement are obtained by projecting the cartography of the transient on the modal basis. In the test presented, the references forced regime of the modal coordinate during the forced period are in gray, the theoretical decaying is in dashed gray, and the actual measurement are in black. 
Because the first mode has a very high quality factor, its transient is much slower compared to the other modes. This interferes with the intended tactile effect, so it is desirable to apply the development previously discussed. In this case, the acceleration factor is 20 (see the spectrogram in fig. 15), which is close to the limit of the method (spill-over). Comparing the growth to the decay, there is a substantial effect for the first mode. Some spillover resulting from the fast dynamic excite the second and fourth mode. There is also some lag on the first mode that is the result of a mismatch of the identified and the actual resonant frequency. Since the quality factor is close to 190 (for comparison the 3rd and 5th modes quality factors are 47 and 63 repectively) a slight error on the resonant frequency (possibly due to variation of the environmental conditions) introduces a phase error. The benefit of the approach is more clear on the spectogram of the velocity at the point of focalisation. The modes are correctly excited; all the modes grow simultaneously until the focusing time at $25 \mathrm{~ms}$. This also shows that for the application the decay -which were left here for demonstration- should also be controlled in practice.

\section{Discussion}

The price of the versatility of the approach is the identification step. However, the results above are encouraging as they demonstrate that, despite the uncertainties on the parameters of the modes and their modal shape, it is possible to realize a given velocity field with an acceptable precision. So the most salient difficulty is the number of modes required which increases the number of uncertainties as demonstrated by fig. 13 but also, makes it more challenging to remain within the voltage limits. This can be addressed by a proper choice of the actual velocity profile and the truncation. For instance, the graphs in fig. 16 depict another example where the pattern is defined as

$$
\dot{w}\left(t_{f}\right)=\left\{\begin{array}{cl}
A \exp \left(-\frac{1}{1-\left(\frac{x-x_{c}}{a}\right)^{2}}\right) & \text { if } x \in\left(-a+x_{c}, a+x_{c}\right) \\
0 & \text { otherwise }
\end{array}\right.
$$

known as the bump function and where $A$ is a scaling factor, $x_{c}$ the center coordinate, and $a$ controls the width. This function has interesting continuity properties and its Fourier transform is rapidly decaying [35]. As mentioned earlier, there exists an analogy between the Fourier transform and the modal projection in this case, hence the result presented in fig. 16 was expected, despite the difference between the actual modes and the 

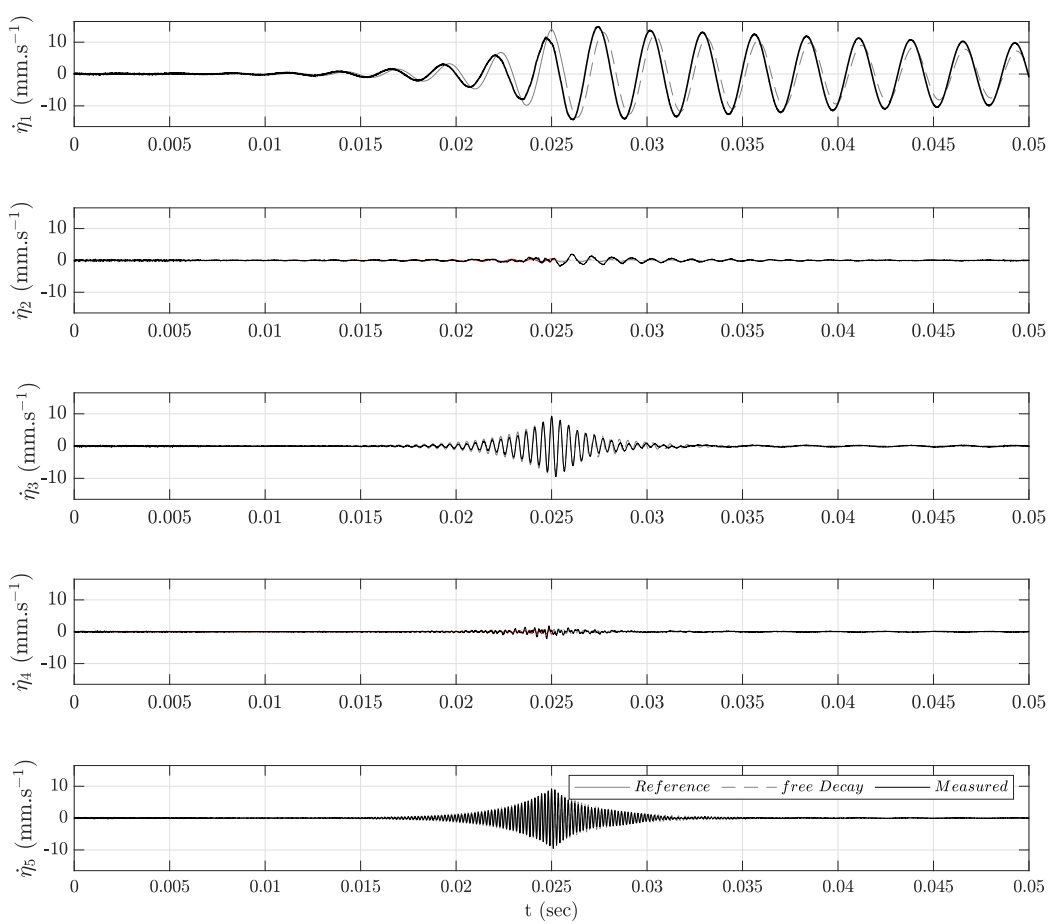

Figure 14: Transients of the five first excited modes used to synthetize a $25 \mathrm{~mm}$ wide sinclike velocity field. The theoretical forced regime (red) is followed by a free decay (green), compared to the actual measured transient obtained by (blue). 


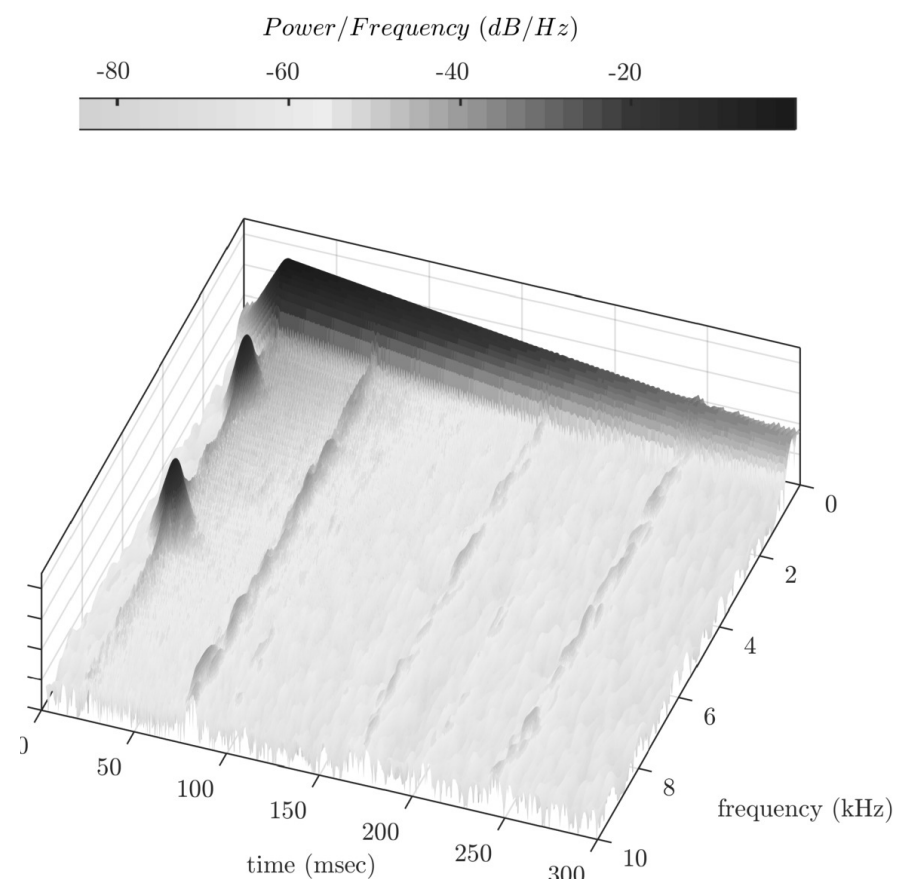

Figure 15: Spectrogram of the velocity measured at the focusing point.

trigonometric functions. The white region shows the projection on modes used for the experimental excitation and the gray region shows the neglected ones based on a distortion criterion (see tab. C.1). Fig. 17 shows experimental results for such truncations. As before, cartographies and modal projections are presented (respectively on the left and right) and show a good agreement between theory and experiment (see tab. C.2).

\section{Conclusion}

This paper has discussed a method to synthesize and approximate velocity field in order to realize a specified velocity field at a given time. The scope of the paper is haptic application where differentiated stimuli are required. Given that such devices are generally realized using plates excited with piezoelectric patches, the equation have been recalled to demonstrate the capacity to excite specifically several modes by a applying well defined voltages to the different ceramics. We have proposed a method to calculate the voltages that must be applied and dispatch them among the piezoelec- 


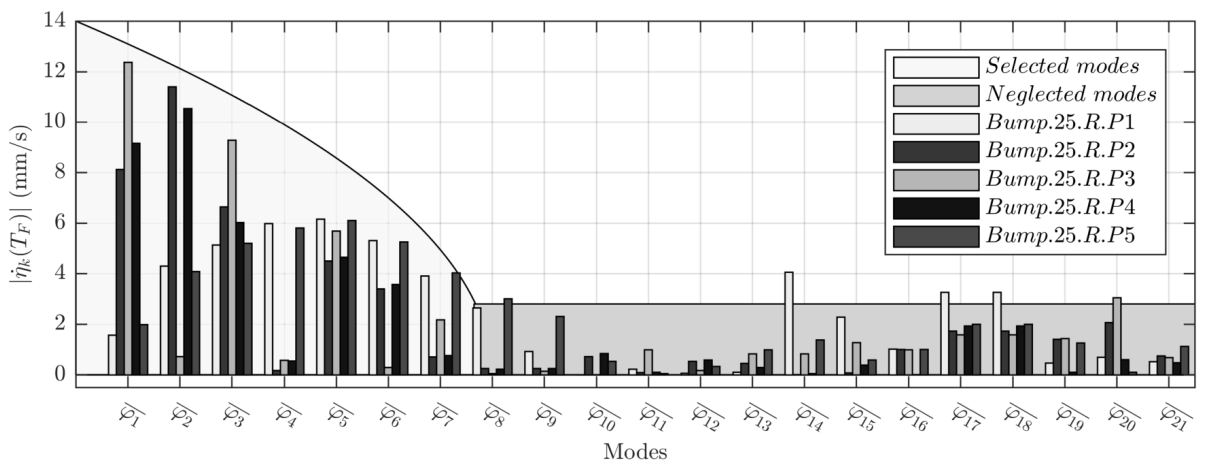

Figure 16: Absolute value of Modal Coefficients of Bump spatial pulse of width $25 \mathrm{~mm}$ pulse width for $x_{c}=-46,-24,0,24$ and $46 \mathrm{~mm}$ ( resp. denoted P1, P2, P3, P4 and P5).

tric patches based on the physical limitation of the ceramics. To do so, the problem is recasted in the modal basis, and the modal forces are specified based on the projection of the projection of the desired velocity field. The advantage of the method is the possibility to generate stimuli independently from the actual location of the actuators and to control the time needed for focusing by properly controlling the rise time for each mode. Experiments have confirmed that it is possible with a reduced number of modes. Several velocity fields have been tested for different velocity fields. Future work will address the problem of parametric variation by introducing a close loop control, and psycho-physical studies to figure out the relevant velocity distribution for haptic applications.

\section{Appendix A. Reduction elements of the plate model}

The model is based on the standard Euler-Bernouilli kinematic assumptions. Since the device is subdivided into domains including either the substrate (index or superscript 0), or the bimorph (index or superscript greater than 0 , within $\left\{1 \ldots N_{b}\right\}$ where $N_{b}$ is the number of the bimorph domain), the different expressions are recalled below. The mass per surface unit are:

$$
\begin{aligned}
& \mathcal{M}_{0}=\rho_{s} h_{s} \\
& \mathcal{M}_{i}=\mathcal{M}_{0}+2 \rho_{p} h_{p} \quad \text { for } i \in\left\{1, \ldots, N_{b}\right\}
\end{aligned}
$$



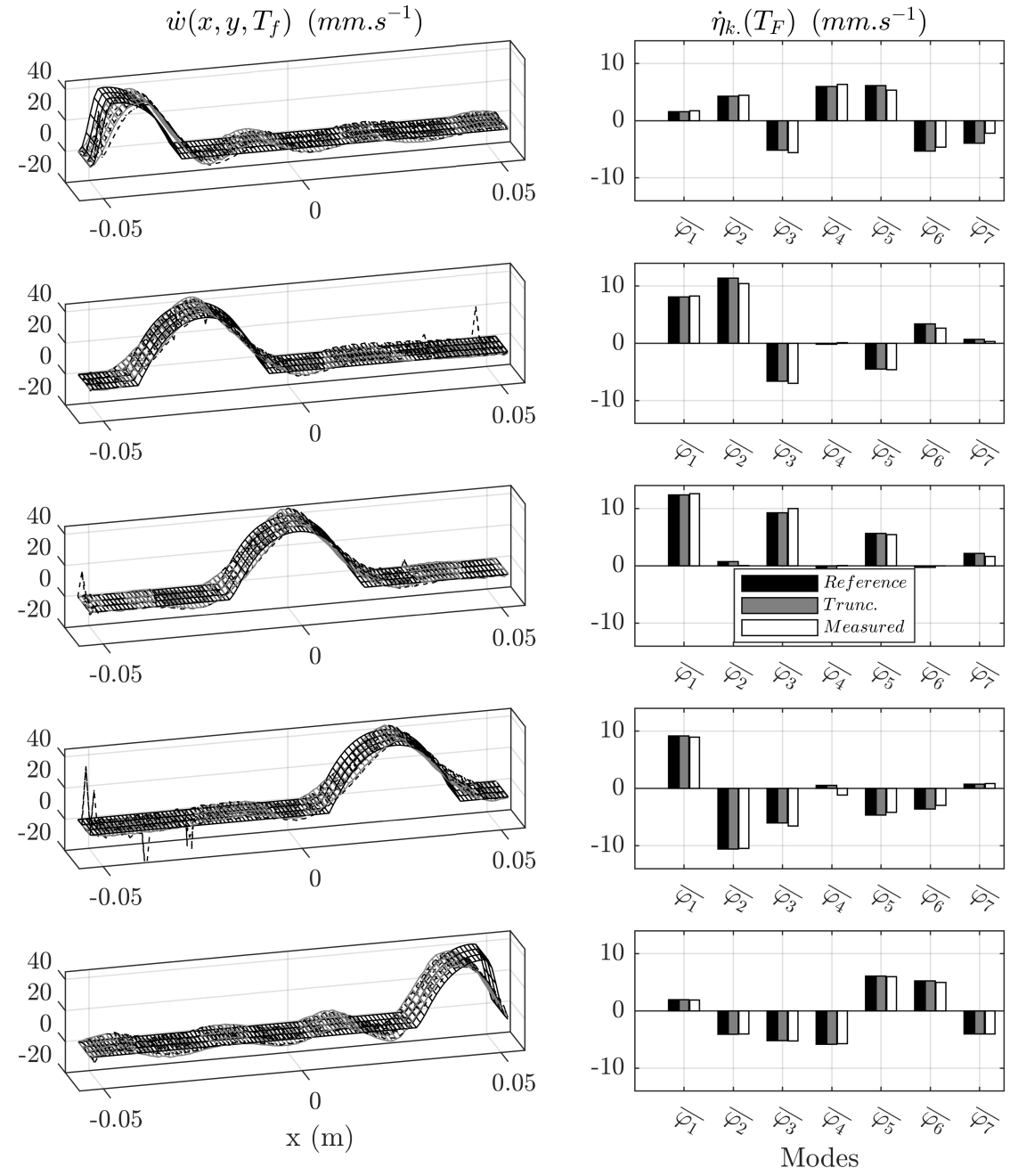

Figure 17: Test results of approximated bump velocity profiles at location points $-46,-24$, 0, 24 and $46 \mathrm{~mm}$, on the left velocity fields for the references, the approached ones and the measured velocities (resp. red, black and blue) and their respective modal projection (right). The truncation includes 7 modes, based on the distortion criteria. 
the moments are given by:

$$
\begin{aligned}
& M_{11}^{i}=\mathcal{D}_{11}^{i} w_{i, 11}+\mathcal{D}_{12}^{i} w_{i, 22} \\
& M_{22}^{i}=\mathcal{D}_{12}^{i} w_{i, 22}+\mathcal{D}_{22}^{i} w_{i, 22} \\
& M_{12}^{i}=\mathcal{D}_{66}^{i} w_{i, 12}
\end{aligned}
$$

with the bending stiffnesses:

$$
\begin{aligned}
& \mathcal{D}_{\alpha \beta}^{0}=c_{\alpha \beta} \frac{h_{s}^{3}}{12} \alpha, \beta=\{1,2\} \\
& \mathcal{D}_{66}^{0}=2 c_{66} \frac{h_{s}^{3}}{12} \\
& \mathcal{D}_{\alpha \beta}^{i}=\mathcal{D}_{\alpha \beta}^{0}+c_{\alpha \beta}^{E}\left(\frac{h_{p}^{3}}{12}+2 d^{2} h_{p}\right) \alpha, \beta=\{1,2\} i \in\left\{1, \ldots, N_{b}\right\} \\
& \mathcal{D}_{66}^{i}=\mathcal{D}_{66}^{0}+2 c_{66}^{E}\left(\frac{h_{p}^{3}}{12}+2 d^{2} h_{p}\right)
\end{aligned}
$$

where $d=\frac{h_{s}}{4}+\frac{h_{p}}{2}$ is distance between the ceramics and the substrate midplane.

\section{Appendix B. Computation of the modal forces}

The procedure to calculate the modal force discussed supposes to specify the transient in the form:

$$
\dot{\eta}_{k}(t)=f_{k}(t) \sin \left(b_{k} t\right)
$$

$f_{k}(t)$ defines the envelop of $\dot{\eta}_{k}(t)$. Presently, a increasing exponential is chosen with the tuning parameters $A_{k}$ and $\gamma_{k}$ :

$$
f_{k}(t)=A_{k} \mathrm{e}^{\gamma_{k} a_{k} t}
$$

Using the Laplace transform, one can write:

$$
\left.\frac{\left(s+a_{k}\right)^{2}+b_{k}^{2}}{s} \mathcal{L}\left[f_{k}(t) \sin \left(b_{k} t\right)\right]=\mathcal{L}\left[\psi_{k}(t)\right)\right]
$$

yielding:

$$
\left.\mathcal{L}\left[\psi_{k}(t)\right)\right]=A_{k} \frac{\left(s+a_{k}\right)^{2}+b_{k}^{2}}{s\left(\left(s-\gamma_{k} a_{k}\right)^{2}+b_{k}^{2}\right)}
$$


So the modal force is:

$$
\psi_{k}(t)=\Psi_{k} \mathrm{e}^{a_{k} \xi_{k} \omega_{k} t}\left[C_{k} \sin \left(b_{k} t\right)+D_{k} \cos \left(b_{k} t\right)\right]+E_{k}
$$

with :

$$
\begin{aligned}
\Psi_{k} & =A_{k} \frac{a_{k}\left(\gamma_{k}+1\right)}{a_{k}^{2} \gamma_{k}^{2}+b_{k}^{2}} \\
C_{k} & =\left(a_{k}^{2} \gamma_{k}\left(\gamma_{k}+1\right)+2 b_{k}^{2}\right) \\
D_{k} & =a_{k} b_{k}\left(\gamma_{k}-1\right) \\
E_{k} & =b_{k} \frac{a_{k}^{2}+b_{k}^{2}}{a_{k}^{2} \gamma_{k}^{2}+b_{k}^{2}}
\end{aligned}
$$

Since in this work $\omega_{k} \Delta t_{k}=\frac{\pi}{2}+2 \pi N_{k}$ is imposed, $A_{k}$ is such that:

$$
\dot{\eta}_{k}^{\star}=A_{k} \mathrm{e}^{\gamma_{k} \xi_{k} \omega_{k} \Delta t_{k}}
$$

Note that the differential equation could be directly used once the envelop is defined.

\section{Appendix C. Experimental results}

In this section, the $\tau$ and $v$ scores of the various shapes tested in this paper are summarized.

\begin{tabular}{lccccc} 
Pulse center & $P_{1}$ & $P_{2}$ & $P_{3}$ & $P_{4}$ & $P_{5}$ \\
\hline Sinc $25 \mathrm{~mm}$ & 0.6831 & 0.9418 & 0.9755 & 0.9359 & 0.7124 \\
Sinc $18 \mathrm{~mm}$ & 0.8833 & 0.9868 & 0.9838 & 0.9887 & 0.9757 \\
Sinc $10 \mathrm{~mm}$ & 0.9521 & 0.9948 & 0.9897 & 0.9928 & 0.9981 \\
Bump $25 \mathrm{~mm}$ & 0.8285 & 0.9727 & 0.9544 & 0.9816 & 0.8717
\end{tabular}

Table C.1: Effect of the truncation: $\tau$-score for the various shapes studied.

\begin{tabular}{lccccc} 
Pulse center & $P_{1}$ & $P_{2}$ & $P_{3}$ & $P_{4}$ & $P_{5}$ \\
\hline Sinc $25 \mathrm{~mm}$ & 0.9739 & 0.9739 & 0.9739 & 0.9739 & 0.9739 \\
Sinc $18 \mathrm{~mm}$ & 0.9792 & 0.9885 & 0.9902 & 0.9611 & 0.9945 \\
Sinc $10 \mathrm{~mm}$ & 0.8771 & 0.9768 & 0.9759 & 0.9386 & 0.9739 \\
Bump $25 \mathrm{~mm}$ & 0.9877 & 0.9953 & 0.9911 & 0.9609 & 0.9973
\end{tabular}

Table C.2: Quality of the focusing : $v$-score for the various shapes studied. 


\section{Acknowledgements}

This work has been carried out within the framework of the project StimTac of IRCICA (institut de recherche sur les composants logiciels et matériel pour la communication avancée).

\section{References}

[1] Seung-Chan Kim, Tae-Hon Yang, Byung-Kil Han, Dong-Soo Kwon, Interaction with a display panel - an evaluation of surface-transmitted haptic feedback, in: 2008 International Conference on Control, Automation and Systems, IEEE, 2008, pp. 278-283.

[2] M. S. Prewett, L. R. Elliott, A. G. Walvoord, M. D. Coovert, A MetaAnalysis of Vibrotactile and Visual Information Displays for Improving Task Performance, IEEE Transactions on Systems, Man, and Cybernetics, Part C (Applications and Reviews) 42 (1) (2012) 123-132.

[3] J. Pasquero, V. Hayward, Tactile feedback can assist vision during mobile interactions, in: Proceedings of the 2011 annual conference on $\mathrm{Hu}-$ man factors in computing systems - CHI '11, ACM Press, Vancouver, BC, Canada, 2011, pp. 3277-3280.

[4] G.-H. Yang, K.-U. Kyung, M. Srinivasan, D.-S. Kwon, Development of quantitative tactile display device to provide both pin- array-type tactile feedback and thermal feedback, in: Second Joint EuroHaptics Conference and Symposium on Haptic Interfaces for Virtual Environment and Teleoperator Systems (WHC'07), IEEE, Tsukaba, Japan, 2007.

[5] A. Agarwal, K. Nammi, K. Kaczmarek, M. Tyler, D. Beebe, A hybrid natural/artificial electrostatic actuator for tactile stimulation, in: 2nd Annual International IEEE-EMBS Special Topic Conference on Microtechnologies in Medicine and Biology. Proceedings (Cat. No.02EX578), IEEE, Madison, WI, USA, 2002-05, pp. 341-345.

[6] O. Bau, I. Poupyrev, A. Israr, C. Harrison, TeslaTouch: electrovibration for touch surfaces, in: Proceedings of the 23nd annual ACM symposium on User interface software and technology, ACM, 2010, pp. 283-292.

[7] A. Yamamoto, T. Ishii, T. Higuchi, Electrostatic tactile display for presenting surface roughness sensation, in: IEEE International Conference on Industrial Technology, 2003, IEEE, Maribor, Slovenia, 2003, pp. 680-684. 
[8] E. Vezzoli, M. Amberg, F. Giraud, B. Lemaire-Semail, Electrovibration modeling analysis, in: M. Auvray, C. Duriez (Eds.), Haptics: Neuroscience, Devices, Modeling, and Applications, Vol. 8619, Springer Berlin Heidelberg, Berlin, Heidelberg, 2014, pp. 369-376.

[9] C. D. Shultz, M. A. Peshkin, J. E. Colgate, Surface haptics via electroadhesion: Expanding electrovibration with johnsen and rahbek, in: 2015 IEEE World Haptics Conference (WHC), 2015, pp. 57-62.

[10] Hui Tang, D. Beebe, A microfabricated electrostatic haptic display for persons with visual impairments, IEEE Transactions on Rehabilitation Engineering 6 (3) (1998) 241-248.

[11] E. Vezzoli, B. Dzidek, T. Sednaoui, F. Giraud, M. Adams, B. LemaireSemail, Role of fingerprint mechanics and non-coulombic friction in ultrasonic devices, in: 2015 IEEE World Haptics Conference (WHC), 2015, pp. 43-48.

[12] T. Watanabe, S. Fukui, A method for controlling tactile sensation of surface roughness using ultrasonic vibration, in: , 1995 IEEE International Conference on Robotics and Automation, 1995. Proceedings, Vol. 1, 1995, pp. 1134-1139.

[13] M. Biet, F. Giraud, B. Lemaire-Semail, Squeeze film effect for the design of an ultrasonic tactile plate, IEEE Transactions on Ultrasonics, Ferroelectrics, and Frequency Control 54 (12) (2007) 2678-2688.

[14] M. Wiertlewski, R. Fenton Friesen, J. E. Colgate, Partial squeeze film levitation modulates fingertip friction, Proceedings of the National Academy of Sciences 113 (33) (2016) 9210-9215.

[15] Xiaowei Dai, J. E. Colgate, M. A. Peshkin, LateralPaD: A surfacehaptic device that produces lateral forces on a bare finger, in: 2012 IEEE Haptics Symposium (HAPTICS), IEEE, Vancouver, BC, Canada, 2012, pp. 7-14.

[16] E. C. Chubb, J. E. Colgate, M. A. Peshkin, ShiverPaD: A glass haptic surface that produces shear force on a bare finger, IEEE Transactions on Haptics 3 (3) (2010-07) 189-198.

[17] M. Amberg, F. Giraud, B. Semail, P. Olivo, G. Casiez, N. Roussel, STIMTAC: A Tactile Input Device with Programmable Friction, in: 
Proceedings of the 24th Annual ACM Symposium Adjunct on User Interface Software and Technology, UIST '11 Adjunct, ACM, New York, NY, USA, 2011, pp. 7-8.

[18] D. J. Meyer, M. Wiertlewski, D. Leonardis, M. A. Peshkin, J. E. Colgate, $[\mathrm{d} 70]$ high-fidelity texture display via programmable friction, in: 2014 IEEE Haptics Symposium (HAPTICS), 2014, pp. 1-1.

[19] W. Ben Messaoud, M.-A. Bueno, B. Lemaire-Semail, Textile fabrics texture: From multi-level feature extraction to tactile simulation, in: F. Bello, H. Kajimoto, Y. Visell (Eds.), Haptics: Perception, Devices, Control, and Applications, Vol. 9775, Springer International Publishing, 2016, pp. 294-303.

[20] S.-Y. Kim, J.-O. Kim, K. Kim, Traveling vibrotactile wave - a new vibrotactile rendering method for mobile devices, IEEE Transactions on Consumer Electronics 55 (3) (2009) 1032-1038.

[21] J.-H. Woo, J.-G. Ih, Vibration rendering on a thin plate with actuator array at the periphery, Journal of Sound and Vibration 349 (2015) 150162.

[22] M. R. Bai, Y. K. Tsai, Impact localization combined with haptic feedback for touch panel applications based on the time-reversal approach, The Journal of the Acoustical Society of America 129 (3) (2011) 12971305.

[23] C. Hudin, J. Lozada, V. Hayward, Localized tactile feedback on a transparent surface through time-reversal wave focusing, IEEE Transactions on Haptics 8 (2) (2015) 188-198.

[24] C. Hudin, J. Lozada, V. Hayward, Spatial, temporal, and thermal contributions to focusing contrast by time reversal in a cavity, Journal of Sound and Vibration 333 (6) (2014) 1818-1832.

[25] E. Enferad, C. Giraud-Audine, F. Giraud, M. Amberg, B. LemaireSemail, Differentiated haptic stimulation by modal synthesis of vibration field, in: 2018 IEEE Haptics Symposium (HAPTICS), IEEE, San Francisco, CA, 2018, pp. 216-221.

[26] L. Meirovitch, Fundamentals of vibrations, internat. ed., [nachdr.] Edition, Mechanical engineering series, McGraw-Hill, Boston, 2008. 
[27] M. Géradin, D. Rixen, Mechanical vibrations: theory and application to structural dynamics, third edition Edition, Wiley, Chichester, West Sussex, United Kingdom, 2015.

[28] H. F. Tiersten, Linear Piezoelectric Plate Vibrations Elements of the Linear Theory of Piezoelectricity and the Vibrations Piezoelectric Plates, Springer US, Boston, MA, 1969.

[29] T. Ikeda, Fundamentals of piezoelectricity, Oxford science publications, Oxford University Press, Oxford ; New York, 1990.

[30] J. A. Mitchell, J. N. Reddy, A refined hybrid plate theory for composite laminates with piezoelectric laminae, International Journal of Solids and Structures 32 (16) (1995) 2345-2367.

[31] P. Heyliger, D. A. Saravanos, Exact freevibration analysis of laminated plates with embedded piezoelectric layers, The Journal of the Acoustical Society of America 98 (3) (1995) 1547-1557.

[32] S. S. Vel, R. Batra, Analysis of piezoelectric bimorphs and plates with segmented actuators, Thin-Walled Structures 39 (1) (2001) 23-44.

[33] A. Benjeddou, M. A. Trindade, R. Ohayon, A Unified Beam Finite Element Model for Extension and Shear Piezoelectric Actuation Mechanisms, Journal of Intelligent Material Systems and Structures 8 (12) (1997) 1012-1025.

[34] A. Deraemaeker, G. Tondreau, F. Bourgeois, Equivalent loads for twodimensional distributed anisotropic piezoelectric transducers with arbitrary shapes attached to thin plate structures, The Journal of the Acoustical Society of America 129 (2) (2011) 681-690.

[35] S. G. Johnson, Saddle-point integration of $C_{-} \infty$ "bump" functions, ArXiv e-printsarXiv:1508.04376. 